\title{
Electron Microscopy Studies Reveal a Physiological Basis of Body Size Determination by TGF- $\beta$ Signaling
}

\author{
R.D. Schultz ${ }^{1}$, E.A. Ellis ${ }^{2}$, and T.L. Gumienny ${ }^{1}$ \\ ${ }^{1}$ Department of Molecular and Cellular Medicine, Texas A\&M College of Medicine, College Station, \\ TX, USA. \\ ${ }^{2}$ Microscopy and Imaging Center, Texas A\&M University, College Station, TX, USA.
}

Body height in mammals is precisely controlled by multiple mechanisms, including long bone growth, transforming growth factor- $\beta$ (TGF- $\beta$ ), insulin, and hormone signaling [1]. In Caenorhabditis elegans (C. elegans), body shape is also influenced by organization of the cuticle (a secreted extracellular matrix comparable to bone) and TGF- $\beta$ signaling $[2,3]$. The best-studied regulator of body size in $C$. elegans is TGF- $\beta$ signaling. Alteration of the DBL-1 TGF- $\beta$ pathway in $C$. elegans generates distinct, dosedependent phenotypes, where long body length correlates with increased signaling and short body length correlates with decreased signaling. The physiological underpinnings of body size differences in $d b l-1$ pathway variants are still unknown. Microarray analyses have determined genes that are up- or downregulated by DBL-1 TGF- $\beta$ pathway signaling, including cuticle collagen genes $[4,5,6]$. In addition, genetic and pharmacological assays indicate that DBL-1 TGF- $\beta$ pathway signaling affect cuticular permeability (Schultz, Bennett, and Gumienny, in preparation). Therefore, we tested the hypothesis that DBL-1 TGF- $\beta$ signaling affects body length through organization of the nematode cuticle.

Using transmission electron microscopy (TEM), we compared the cuticles of animals expressing different levels of DBL-1. We examined subcellular components of the cuticle in wild-type animals, small animals lacking DBL-1 $(d b l-1(-))$, and long animals overexpressing DBL-1 $(d b l-1(++))$. As the cuticle of $C$. elegans is extremely tough, we optimized methods for sample preparation using the PELCO BioWave ${ }^{\circledR}$ Pro microwave. We preserved and stained the cuticle lipids by adding $0.2 \%$ (wt/vol) malachite green as an additive to the fixative, because lipids are normally stripped from specimens during standard fixation procedures [7]. To provide more contrast, specimens were post-fixed in $1 \%$ (wt/vol) osmium tetroxide with $1.5 \%(\mathrm{wt} / \mathrm{vol})$ potassium ferricyanide and then stained en bloc with $0.5 \%$ aqueous uranyl acetate. Finally, the samples were dehydrated, suspended in propylene oxide, infiltrated, and embedded. Gold sections were post-stained with $2 \%(\mathrm{wt} / \mathrm{vol})$ aqueous uranyl acetate followed by Reynolds lead citrate [8]. Five or six animals of each genotype were examined.

We found that differences in DBL-1 pathway signaling correlate with changes in composition and organization of the cuticle. In wild-type animals, an outer layer of lipids binds malachite green (Figure 1). Long animals overexpressing DBL-1 have a thicker layer of malachite green bound to the surface coat (Figure 2), while small animals lacking DBL-1 have very little bound malachite green (Figure 3). Further, malachite green preserves and differentially stains the inner layers of cuticle, clearly distinguishing the cortical, medial, and basal layers and their components (Figures 1-3). Subcellular analysis reveals that DBL-1 affects both the medial and basal layers of the cuticle. $d b l-1(-)$ animals (Figure 3) have a strikingly denser medial layer compared to wild-type (Figure 1) and $d b l-1(++)$ animals (Figure 2). We found the composition of the topmost basal layer in animals overexpressing DBL-1 (Figure 2) is altered compared to either wild-type (Figure 1) or dbl-1(-) animals (Figure 3). These results show that body size changes caused by differences in DBL-1 TGF- $\beta$ pathway signaling levels are associated with structural variation of the cuticle. 


\section{References}

[1] R Visser et al, Pediatric Endocrinology Reviews 6 (2009), 375.

[2] JM Kramer et al, Cell 55 (1988), 555.

[3] C Savage-Dunn in "WormBook," ed. The C. elegans Research Community.

[4] M Mochii et al, Proc Natl Acad Sci USA 96 (1999), 15020.

[5] J Liang et al, Dev Biol 305 (2007), 714.

[6] AF Roberts et al, BMC Developmental Biology 10 (2010), 61.

[7] RJ Teichman et al, Biology of Reproduction 7 (1972), 73.

[8] ES Reynolds, The Journal of Cell Biology 17 (1963), 208.

[9] This work was supported by NIH 1R01GM097591-01 and by TAMHSC MCMD start-up funds.

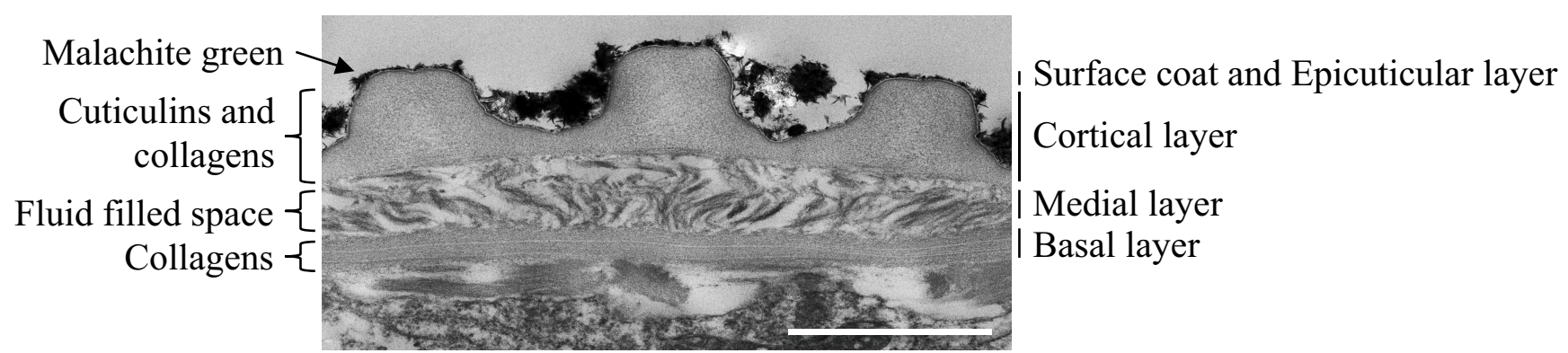

Figure 1. The cuticle of a wild-type animal stained with malachite green, which binds to lipids on the outer layer of the cuticle. Cuticular components are shown on the left, while cuticular layers are presented on the right. Scale bar $=1 \mu \mathrm{m}$.

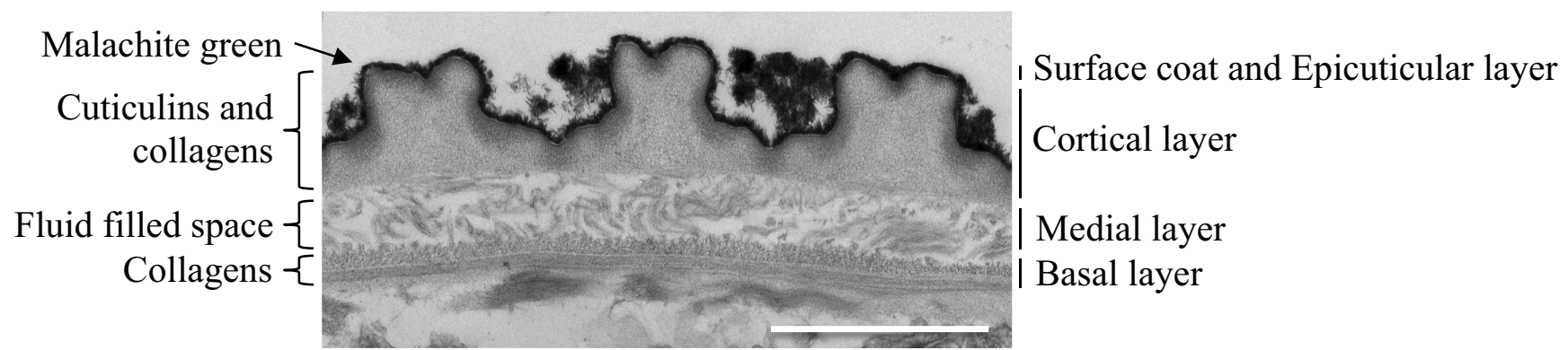

Figure 2. The cuticle of a $d b l-1(++)$ animal, which has altered medial and basal layers and an increased amount of malachite green bound to lipids in the surface coat of the cuticle. Scale bar $=1 \mu \mathrm{m}$.

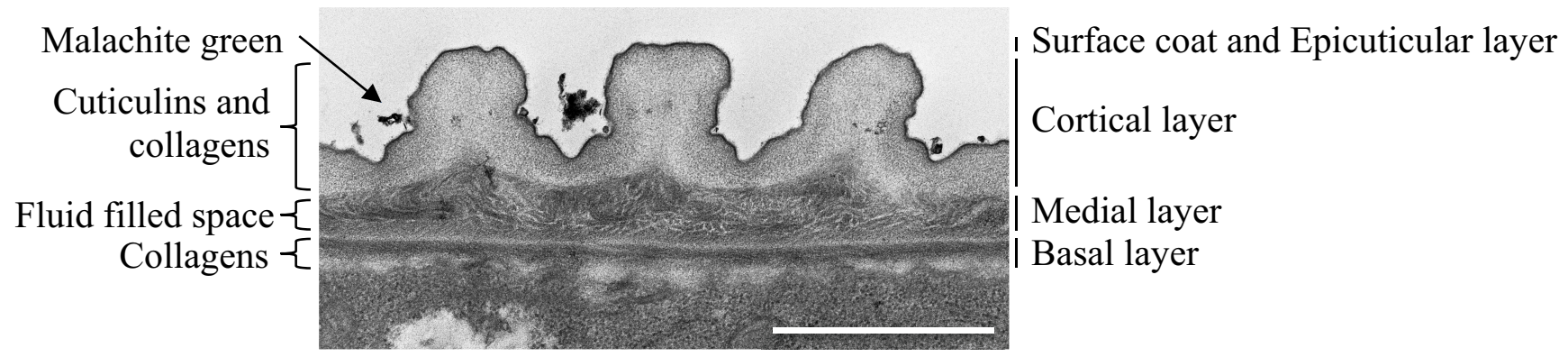

Figure 3. The cuticle of a $d b l-1(-)$ animal, which has a dense medial layer and little malachite green bound to lipids in the cuticular surface coat. Scale bar $=1 \mu \mathrm{m}$. 